\title{
Study on the Profitability of Fenjiu Group Based on the Financial Index System
}

\author{
Yifan $\mathrm{Niu}^{1, \mathrm{a}, \uparrow}{ }^{\dagger}$, Zhaoqi Zhou ${ }^{2, \mathrm{~b}, \uparrow}$, and Zhuoran $\mathrm{Wan}^{3, \mathrm{c}, \uparrow}$ \\ ${ }^{1}$ International Education College, Hebei Finance University, 071051 Hengxiang North Street 3188, Baoding, China; \\ ${ }^{2}$ Huazhong University of Science and Technology, School of Management, 430074 Luoyu Road 1037, Wuhan, China; \\ ${ }^{3}$ Sichuan International Studies University, School of Management and Business, 400031 Zhuangzhi Road 33, \\ Chongqing, China. \\ a632963736@qq.com, ${ }^{b} U 201815924 @$ hust.edu.cn, ${ }^{c} 20201936150056 @$ stu.sisu.edu.cn \\ * Corresponding author: U201815924@hust.edu.cn \\ These authors contributed equally.
}

\begin{abstract}
In recent years, the Baijiu industry develops rapidly and contributes to China's GDP. However, with the market competition becoming more and more fierce, the Baijiu industry may be impacted. Facing the potential risk, the Baijiu industry has to improve the profitability to fit the changeable market. Fenjiu Group's micro and macro analysis results are presented in this paper as an example. Literature research method, case analysis method, and experiential summary method are taken for deeper research. Through the calculation of Fenjiu Group's financial index, Fenjiu Group's gross profit margin showed a small fluctuation from 2015 to 2018. Fenjiu Group's ROTA declined from $13.2 \%$ to $12.8 \%$ between 2018 and 2019, indicating the growth of profit can't match the recent expansion of Fenjiu Group's asset size. In terms of investor profitability, Fenjiu Group's ROE grew from $11.8 \%$ to 26\% between 2015 and 2019. According to the above analysis, some suggestions are put forward to the existing problems, such as brand diversification propaganda, optimizing the capital structure, broadening financial channels, and controlling the expansion of asset size. The research results of this paper hope to help Fenjiu Group improve better and enhance the business value in the future.
\end{abstract}

Keywords: Shanxi Fenjiu, Profitability, Problem, Strategy.

\section{INTRODUCTION}

China is the main production area of Baijiu in the world, and at the same time, baijiu industry really contributes a lot to China GDP, the production amount and sale of Baijiu in China is really considerable, the production amount is 1.23 million kiloliters in just February 2021, with the rate of accumulated increase of $18.9 \%$, and the sales-output ratio in recent years is always more than $98 \%$. Meanwhile, the rivalry in the Baijiu industry is going to be more and more fierce due to related products both domestic and abroad, the number of Baijiu companies above the designated scale had decreased from 1423 in 2013 to 1176 in 2019, which means the concentration of this industry has been going up. Doing research in the Chinese Baijiu industry is of great meaning, on the one hand, we can have a deep insight into the past, current, and future situation of this traditional Chinese industry. In China, compared with companies in other industries, Baijiu companies not only have steady performances during recent years but also bear the weight of national confidence. On the other hand, whether the profitability of Baijiu companies can satisfy their stakeholders still remain a serious question. And by analyzing the profitability of Baijiu, we may get the answer to this question.

Many researchers have done various researches on the Baijiu industry and the profitability of Baijiu companies. Jin found that the prohibition of Baijiu had led to a sharp decrease in profit rate in the whole industry, especially the high-end brands. Moreover, during the transformation period between 2013 and 2016, the transforming strategy of various Baijiu companies was not well executed. Even though the profitability of Baijiu companies had recovered to a certain extent, the situation was still unsatisfactory[1]. Wen has discovered that the market has similar effects on different Baijiu companies. From 2017, the whole 
industry of Baijiu experienced an increase of price and sales amount at the same time, especially for the midto-high-end brands[2]. Thus by comparing the performance of a Baijiu company with the average level of the Baijiu industry, we can easily judge the performance of a single company. Zhu found that in our "Big Data Era", the budget management of Baijiu companies was lag behind, there were many aspects such as system regulation and human resource need to be strengthened and progressed [3]. Therefore, it is obvious that the Baijiu industry is a special industry, it is greatly influenced by government policies, and it also has its own operating problems and has great progress space. A great number of researchers have mainly researched the effects of policies, marketing, and inter operating on Baijiu industries. The impacts from the macroeconomic environment both domestic and abroad on the Baijiu industry are still insufficient. Worldwide economic situation and social culture will greatly influence the profitability of Baijiu companies (eg. COVID-19).

As the above says, we will take Fenjiu Group to do its micro and macro analysis. We will then analyze its financial index to better understand its current situation and find out the existing problems. In combination with the problems, we will put forward some proposals that might be helpful.

\section{METHODOLOGY}

This paper focuses on analyzing the profitability of Fenjiu Group, and we used the literature research method, case analysis method, and experiential summary method as our research methods. Next, I will introduce these three research methods in detail.

\subsection{Literature research method}

The literature research method mainly refers to the method of collecting, discriminating, and sorting out literature and forming a scientific understanding of the facts through the study [4]. In this paper, first of all, through consulting the published journals and papers on Chinese and foreign academic websites to find out the problems existing in the development process of the Baijiu industry, especially Fenjiu Group. Then find out the research gaps in this area to focus on analysis, put forward problems, and determine the specific research topic. Secondly, search the official website of Shanghai Stock Exchange, Sina Finance website, National Bureau of Statistics, and other websites to collect materials and data related to the research topic (e.g., the annual report of the company), to provide data and information sources for this paper. Then, find the calculation method of the company's profitability index from the relevant books and calculated it according to the data collected above. Finally, combine with the industry report of the Baijiu industry, understand the development model and development prospect of the Baijiu industry in China, and put forward corresponding solutions for the problems studied in this paper.

\subsection{Case analysis method}

The case analysis method is a scientific analysis method that makes a thorough and careful study of representative things or phenomena so as to obtain an overall [5]. By analyzing some case in the Baijiu industry, it can help us to deepen understanding of the current situation of the development of Baijiu-making industry, to obtain more comprehensive information about the industry and better analyze it the problems in the development of the company provides guidance and help. In this article, we will choose Fenjiu Group as our research object. We will cite the case of Jiang Xiaobai in marketing to young people, help us better analyze and solve the problems in the development process of Fenjiu Group.

\subsection{Experiential summary}

The experiential summary method is a method to systematize and theorize practical activities by summarizing and analyzing the specific situations in practice and then turn them into experience [6]. As far as this paper is concerned, all useful information can be summarized by collating and analyzing literature reviews, enterprise data, and industry information. Use the profitability index data of other Baijiu companies in the industry compared with Fenjiu Group and analyze the results. Based on the development experience of other enterprises in the Baijiu industry and their operation and marketing methods, according to the actual demand and development status of Fenjiu Group to put forward some corrective measures and suggestions that can promote its further development.

\section{RESULT}

\subsection{Analysis of the environment of Fenjiu Group}

\subsubsection{Analysis on macro-environment}

Fenjiu Group mainly faces the Chinese mainland market. In terms of policy, the impact of prohibition has been very small in recent years. And in recent years, relevant policies have focused on industry standardization, and the overall policy environment is relatively moderate, meanwhile, the high-speed development of the economy has promoted people's purchasing power, especially for high-grade Baijiu. At the same time, the improvement of production, storage, and logistics technology has reduced the cost rate of the Baijiu industry, however, it also increases consumer 
choice, resulting in increased competition in the Baijiu industry, and with the upgrading of social health concepts, people tend to drink less alcohol, which will impact on the Baijiu industry as a whole. Overall, the macro-environment is with both opportunities and challenges.

\subsubsection{Analysis on micro-environment}

Firstly, Shanxi Fenjiu is an important part of domestic famous Baijiu and Baijiu culture. Its historical origin and popularity give it a major competitive advantage. It has two famous brands, "Xinghua Village" and "Zhuyeqing", "Xinghuacun" has a brand value of more than 5 billion yuan, which is the most valuable 50 brands in China[7]. "Zhuyeqing" is the only famous health wine in China recognized by the Ministry of Health. Then, Shanxi Fenjiu's natural geographical conditions are superior, brewing technology superb, unique taste, good reputation, these are its robust opportunities. However, there are still deficiencies in marketing and product diversification, the monotony of the products makes it difficult for Shanxi Fenjiu to develop in the young market, and the lack of marketing makes Shanxi Fenjiu not well promoted in the southern market. And the rivalry with famous Baijiu companies like KWEICHOW MOUTAI GROUP, WULIANGYE is very fierce, and wine, medicinal wine, and cheap Baijiu also have a great potential threat to Fenjiu Group.

\subsection{Profitability status quo and existing problems of Fenjiu Group}

This paper will analyze the profitability of Fenjiu Group in recent five years from the enterprise's sales profitability, asset profitability, and investor profitability.

The financial indicators used are operating profit rate, gross profit margin, return on total assets, return on equity, and earnings per share.

\subsubsection{Analysis of sales profitability}

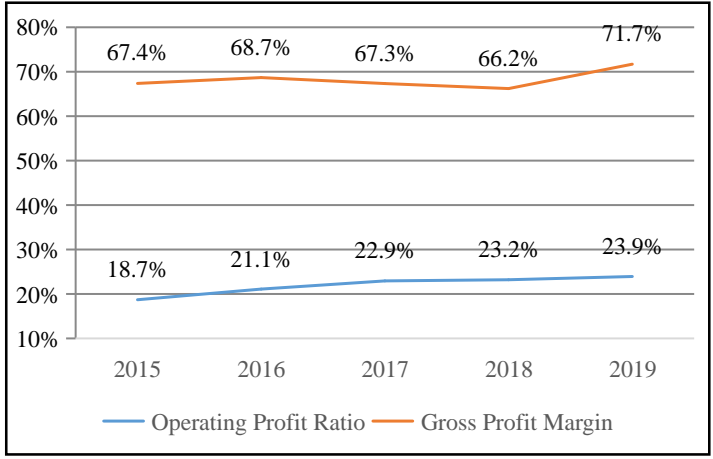

Fig. 1. Sales profitability of Fenjiu Group

The operating profit ratio reflects a business manager's ability to earn profits from operations and take into account operating costs simultaneously [8] Gross profit margin is usually referred to as gross profit as a percentage of net [9]. And the increase in gross profit margin can reflect the improvement of corporate profitability. From figure 1, we can see that the operating profit ratio of Fenjiu Group increased from $18.7 \%$ to $23.9 \%$ which showed an upward trend during the five years between 2015 and 2019. It showed that the company's ability to create profits through business operations was strengthening. In addition, in terms of gross profit margin, the gross profit margin of Fenjiu Group fluctuated at about $67 \%$ from 2015 to 2018 and then increased to $71.7 \%$ in 2019 . In recent years, the company has increased the development of the national market and the amount of advertising, efforts to enhance its brand influence. In 2019, the rising trend of gross profit margin reflected the company's achievements in product sales and brand image construction. Besides, the operating income increased by $25.79 \%$ compared with 2018 , which increased more than the operating cost. Therefore, these two rates increased in 2019. Fenjiu Group, as a whole, reflects their good sales profitability.

\subsubsection{Problems with sales profitability:}

The ability of enterprise cost control needs to be noticed and improved. In recent years, Fenjiu Group has been committed to exploring the international market, promoting brand construction, and enhancing its market influence. Implementing a series of activities successfully helped the enterprise expand the sales channels so that the company's market competitiveness and profitability continue to enhance. But it is worth noting that in the process of promoting brand building, it is also necessary to control the cost. The main reason for the slight fluctuations in the company's gross profit margin between 2015 and 2018 is the increase in operating costs. Among them, the increase in enterprise sales expenses and research and development expenses directly leads to an increase in operating costs.

\subsubsection{Analysis of asset profitability}

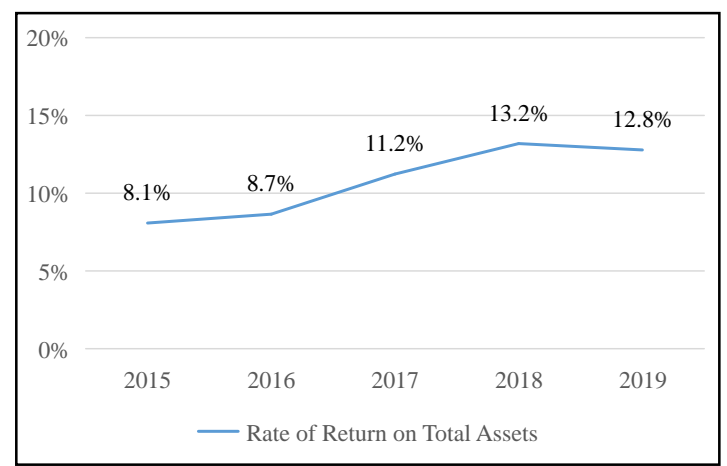

Fig. 2. Asset Profitability of Fenjiu Group 
The rate of return on total assets refers to the overall profitability of all the assets of an enterprise, including net assets [10]. It can reflect the operating conditions of the assets of an enterprise. It is an important indicator to evaluate the operating benefits of the assets of an enterprise. As shown from the above figure, the return rate of Fenjiu Group's total assets went up from $8.1 \%$ to $13.2 \%$ between 2015 and 2018, following a slight decline in 2019. It indicates that the utilization efficiency of the company's assets is getting worse, the profit created by using assets is shrinking, and the profitability of assets is declining.

\subsubsection{Problems with asset profitability:}

The utilization efficiency of enterprise funds is low. From 2015 to 2018, the net assets of enterprises have maintained a growth rate of about $55 \%$, with a large increase. In 2019, the company's net profit increased by $28.63 \%$, a smaller increase which is lower than in former years. At the same time, corporate assets are growing continuously. Therefore, the decline of the company's net profit growth and total assets increase directly lead to the decline of the return rate on total assets. And the main reason for the decline is that the scale of assets continues to expand but doesn't bring the corresponding scale of profit.

\subsubsection{Analysis of investor profitability}

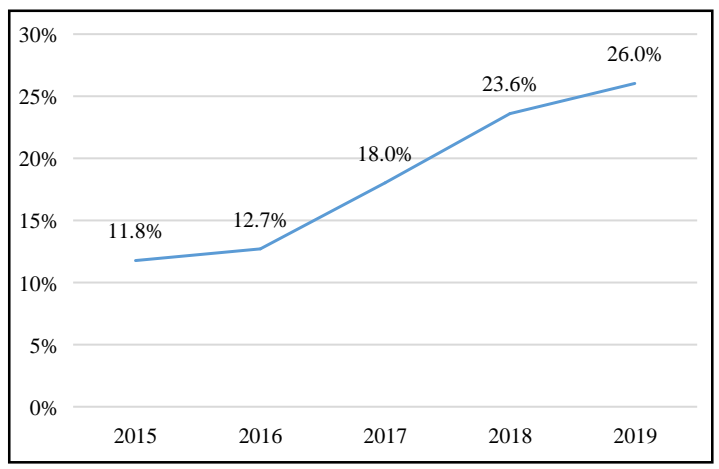

Fig. 3. ROE of Fenjiu Group

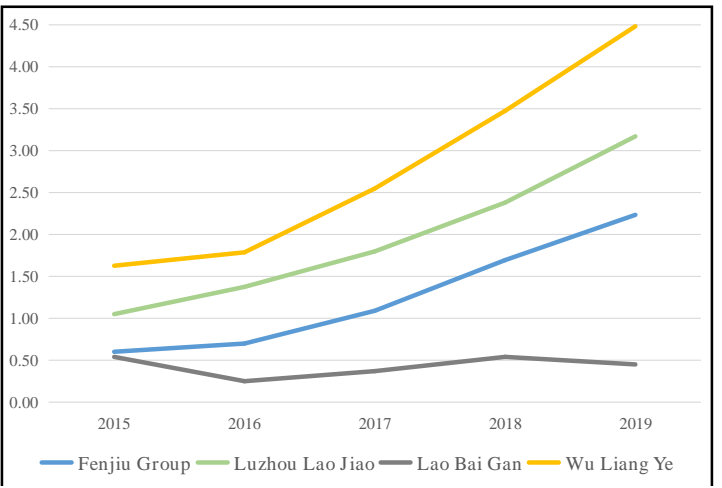

Fig. 4. EPS of Fenjiu Group, Luzhou Lao Jiao, Lao Bai Gan, and Wu Liang Ye
Return on equity (ROE) reflects the income level of shareholders' equity and can be used to measure a company's efficiency using its own [11]. The higher the index value, the higher the return on the investment. Earnings per share (EPS) is the company's net profit divided by the number of common shares issued, is a core index to measure the profitability of corporate [12]. The higher a company's EPS, the more profitable the company is perceived by investors. As can be seen from the changing diagram of the company's ROE in Figure3, although the growth rate of Fenjiu Group's ROE has slowed down from 2018 to 2019 compared with the previous years, it has still maintained an upward trend that the ROE of Fenjiu Group grew from $11.8 \%$ to $26 \%$. And in Figure4, the blue line represents data related to the Fenjiu Group. The company's EPS has also been increasing, indicating that the company's investment income is constantly improving, and the enterprise's profitability in this respect has improved.

\subsubsection{Problems with investor profitability:}

The overall performance is good, but there is still room for improvement. The increase of enterprise return on equity may be due to the improvement of enterprise income level and should take into account the proportion of the company's borrowed funds in assets and its influence on the ratio. From the development of Fenjiu Group itself, the profitability of its investors is in line with the trend of market development. However, compared with other companies in the same industry, Fenjiu Group still has great room for progress and great growth potential.

\section{DISCUSSION}

\subsection{Fenjiu Group needs to strengthen the cost management of market development}

\subsubsection{International market development and cost management}

With the development trend of economic globalization, the acceptance of Chinese Baijiu in the world is getting higher and higher, and there are more and more Baijiu products on overseas shopping websites, but relative to the huge volume of the international market, the existing international market development degree is far from enough, with such an supportive market environment. It is important to invest heavily in market development, but the cost of full roll-out is too great. The key to opening up the international market lies in overseas marketing and pioneer product selection. The promotion of highquality "star products" and low-cost "entry products" should be given special attention, it is very important to establish a positive image of high-end while quickly 
entering the market with low-priced products to improve the international market visibility. In addition, the R\&D of Baijiu products which suits Western people's tastes is also be possible to quickly open the international market.

\subsubsection{Develop domestic market of young people and cost management}

In China, related data shows that the main target group of Baijiu is still the middle-aged and old people, and the young people's Baijiu market has great development potential. In order to open up the youth market, Fenjiu Group needs to get rid of the stereotype of "old-fashioned" "high-grade" "expensive vintage" and actively develop low-alcohol and low-price beverage-type Baijiu to meet the needs of young people, in this respect can learn from "Jiang Xiao Bai" product design. In the cost management aspect, it must avoid the low performance-to-price ratio advertisement expense positively, and chooses the more effective propaganda way. At the same time, sales channels also need to be further developed. The main sales channel of Fenjiu Group is still large-scale merchants. Strengthening the promotion of online sales channels and the development of diversified channels can promote the development of the young people's market.

\subsection{Brand Diversification Propaganda}

To develop the Chinese and foreign markets further, Fenjiu Group has been implementing the strategy of developing the emerging market. Still, the effect is not obvious enough, so Fenjiu Group has to advertise its products in diversified areas. Fenjiu Group needs to ensure the quality of their products is great. Then they can consult with celebrities to get endorsements or sponsor a variety show to make more exposure for this brand that they can attract more consumers. Meanwhile, they can make the advertisements more creative and enrich the content of advertisements to fit the consumer psychology that will enhance product competitiveness in the industry. They can also organize some visitors to visit their factories, on the one hand, the visitors can have a better understanding of the productive process and the quality of the products. On the other hand, this kind of activity can arouse the visitors' interest to attract consumers to buy the products. Besides the offline propaganda, Fenjiu Group can conduct big data analysis among the consumers on the Internet and they can make digital advertisements.

\subsection{Optimize the Capital Structure}

\subsubsection{Cost Control}

In recent years, the selling and scientific research costs increased a lot due to their strategy of developing the new market, so Fenjiu Group needs to control the cost well for better development in the future. Fenjiu Group can reduce offline publicity moderately, shifting the marketing emphasis on online propaganda, which can broaden the marketing channel and reduce the selling expenses. At the same time, Fenjiu Group is supposed to plan their future research aim and interests reasonably to avoid wasting the scientific research funds to can control the research cost.

\subsubsection{Broaden Financial channels}

Fenjiu Group has built a great reputation among the investors, and the profitability of their investors is good as the ROE of Fenjiu Group went up from $11.8 \%$ in 2015 to $26 \%$ in 2019 and the EPS increased steadily. However, the financial channels of Fenjiu Group are relatively unitary, so they can broaden their financial channels to improve profitability.

\section{(1) Mortgage Liquor Base for Funds}

As recorded in the annual report, Fenjiu Group aims to expand the liquor base's capacity, making them an opportunity to mortgage liquor base for funds that will make the capital turnover flexible, and Fenjiu Group can have more funds to allocate in the short term. As a result, they can promote the development of this brand's products.

\section{(2) Issue Commercial Paper}

Fenjiu Group can accumulate capital by issuing commercial paper moderately. Fenjiu Group will gain great capital in the short term with low cost, and the awareness and reputation of Fenjiu Group's products will be improved as well. A lot more potential investors may also be attracted.

\subsection{Control the Expansion of Asset Size}

Through the decrease of Fenjiu Group's net profit growth rate in 2019, we can see from the growth of profit can't match the expanding asset size of Fenjiu Group in recent years, so Fenjiu Group needs to control the growth rate of their expanding asset size within limits. Fenjiu Group is supposed to expand the asset size progressively rather than blindly if Fenjiu Group will face the risk of the capital chain. For example, LETV blindly expanded the asset size in the rising period, which leads the capital chain to break and make LETV go into liquidation.

\section{CONCLUSION}

By analyzing the macro and micro environment, we know that with the continuous development of the 
Baijiu industry, more and more Baijiu brands flood into the market, intensifying the industry rivalry. In addition, the continuous optimization and innovation of other liquor varieties and the constant changes of social drinking culture also have a certain impact on the development of Baijiu enterprises. For Fenjiu Group, after we analyze its profitability based on financial indicators, we believe that the enterprise mainly has the following three problems in its development process. The first one is that Fenjiu Group, in the profitability of its sales, the enterprise's cost control is not good enough. Second, in terms of the profitability of their assets, the capital utilization rate of enterprises is low. Third, compared with other companies in the industry, Fenjiu Group still has a lot of room to make progress in terms of their investors' profitability. First of all, Fenjiu Group should optimize the capital structure, broaden financing channels, appropriate part of its offline marketing into online marketing to save expenses. Besides, enterprises should also properly control the expansion of their assets in order to prevent the rupture of the capital chain. Besides, Fenjiu Group should pay more attention to the diversified publicity of their brands and increase their exposure by participating in or holding different types of activities to attract more potential consumers. And implement different development strategies for the Chinese market and the international market to deepen people's impression of Fenjiu Group. Given the above problems, we put forward some suggestions to help Fenjiu Group improve its profitability.

However, this paper also has some one-sidededness and limitations when analyzing the profitability of Fenjiu Group. This paper adopts three research methods: the literature method, case analysis method, and summary method. In the process of use, most of the references we refer to contain the subjective judgment of the authors, which may affect our views. Besides, the situation of inexact case analysis and incomplete summary may happen. Apart from that, the analysis of the profitability of Fenjiu Group in this paper is only based on the profitability index of financial indicators, without considering other financial indicators. Nevertheless, the profitability of an enterprise is not only affected by financial indicators, but also by other non-financial factors. Therefore, when analyzing the research topic, it is necessary to combine various research methods and consult various materials to conduct a more comprehensive and in-depth analysis of the research object.

\section{REFERENCES}

[1] Jin, China Commercial Forum, Research on the influence of prohibition on the profitability of Baijiu enterprises
[2] Wen, Fortune times, Analysis on the profitability of Baijiu-listed companies: A case study of Hengshui Laobaigan, 09, 145 (2020)

[3] Zhu, Commercial message, Research on the influence of prohibition on the profitability of Baijiu enterprises, 18, 115 (2020)

[4] G. Lin, 'Higher Education Research MethodologyLiterature Method'. International Education Studies, 2(4), p179

[5] S. Crowe, 'The Case Study Approach'. BMC Medical Research Methodology, 11(1), p100. (2011)

[6] Experiential summary method_360 Encyclopedias.

[7] Jiang, Brewing Technology, Fenjiu was listed in the top 50 of "Best Chinese brand value ranking 2013", 12, 24, (2013)

[8] Corporate Finance Institute. Operating Profit Margin - Learn to Calculate Operating Profit Margin.(2021)

[9] A. Bloomenthal, Why Gross Profit Margin Matters. Investopedia.

[10]Rate of return on total assets_360 Encyclopedias.

[11] J.\&O. Fern, How Return on Equity (ROE) Works. Investopedia.

[12] J.\&O. Fern, Earnings Per Share (EPS). Investopedia. 